\title{
Diagnose and Prediction of Different Machine Learning methods for Knee Osteoarthritis: a protocol for systematic review and meta-analysis
}

yu zhou ( $\nabla$ zhouyuyu105@126.com)

Beijing Chaoyang Hospital

\section{Tong Mu}

Department of Stomatology,Emergency general hospital,

\section{Xiaochuan Kong}

Department of Orthopedics,Beijing chaoyang hospital, Capital medical university

\section{Le Zhang}

Department of Orthopedics, Beijing-chaoyang hospital, Capital medical university

\section{Protocol}

Keywords: knee osteoarthritis, machine learning, Artificial intelligence, diagnose, prediction

Posted Date: September 20th, 2021

DOI: https://doi.org/10.21203/rs.3.rs-903246/v1

License: (c) (i) This work is licensed under a Creative Commons Attribution 4.0 International License. Read Full License 


\section{Abstract}

Background: Knee osteoarthritis $(\mathrm{OA})$ is a chronic and progressive joint disease with a higher contributor to global disability, mainly in the elderly and particularly in women. The available diagnostic approaches such as $\mathrm{X}$-ray, computed tomography and magnetic resonance imaging have large precision errors and low sensitivity. Machine learning $(\mathrm{ML})$ is the application of probabilistic algorithms to train a computational model to make predictions, it has great potential to become a valuable clinical diagnostic tool. This review aims to determine the diagnosis and prediction accuracy of different machine learning methods for Knee Osteoarthritis

Methods: Two reviewers systematically searched Cochrane, PubMed, EMBASE, and Web of Science (last updated in June 2020) for eligible articles. To identify potentially missed publications, the reference lists of the final included studies were manually screened. Outcomes assessed were test characteristics such as accuracy, sensitivity, specificity, and area under the receiver operating characteristic curve (ROC). We will use the Quality Assessment of Diagnostic Accuracy Studies (QUADAS-2) tool to assess the risk of bias and applicability. Two independent reviewers will conduct all procedures of study selection, data extraction, and methodological assessment. Any disagreements will be consulted with a third reviewer. RevMan 5.3 software and Stata V15.0 will be used to pool data and to carry out the meta-analysis if it is possible.

Results: This systematic review will provide a high-quality synthesis of machine learning for diagnose of knee Osteoarthritis from various evaluation aspects including accuracy, sensitivity, specificity and AUC.

Conclusion: The findings of this systematic review will provide latest evidence of diagnosis and prediction of different machine learning for patients with knee Osteoarthritis.

Ethics and dissemination: No individual patient data will be used in this study; thus, no ethics approval is needed.

\section{Systematic review registration: PROSPERO CRD: 42019133305}

\section{Introduction}

Knee osteoarthritis (OA) is the most common musculoskeletal disorder, Mainly in the elderly population and particularly in female ${ }^{1}$. With a global prevalence of radiographically confirmed symptomatic knee $O A$ estimated to be $3.8 \% 2,3$ and a lifetime risk of symptomatic knee OA in Western populations estimated to be over $40 \%^{3}$. The annual incidence of knee OA in the United States is estimated at 240 persons per $100,000^{4}$. The report shows that patients with knee OA have an almost two-fold increased risk of sick leave and about $40-50 \%$ increased risk of disability pension compared with the general population ${ }^{5}$. Over time, it has caused large economic burden on individual and society. At present, the aetiology and pathology of this disease are not clear, there has no effective cure for knee $O A$, and only control of the 
symptoms and treatment to prevent further development of the disease are available. ${ }^{6-8}$. Thus, reliable and early detection of knee $\mathrm{OA}$ are particular important to conquer this disease.

In the past few decades, the diagnosis of knee OA in the clinic is most often made using the 1986 criteria of the American College of Rheumatology. These criteria include a combination of the patient's age, signs and symptoms on physical exam, radiographic and/or laboratory evidence ${ }^{9}$. In clinical, taking X-ray images of the diseased knee is the most commonly method used for the diagnosis of OA, but it is not effective in the early period of etiologic changes since X-rays only provides an approximation of the articular cartilage, and visible joint narrowing and osteophytes typically manifest in the later stages of $\mathrm{OA}$. Over the past decades, Magnetic resonance imaging (MRI) as a non-invasive imaging modality that can be used to establish knee OA diagnosis, assess disease severity, and monitor disease progression. However, the studies have shown that have moderate sensitivity of $61 \%$ and high specificity of $82 \% 10$, and fail to deliver a cost effective, simple to use, and suitable solution for mass screenings and repeated use $^{11,12}$. The diagnosis of knee $O A$ is still a challenge in the biomedical engineering and different methods need to be explored.

Machine learning (ML) is a form of artificial intelligence, and is the application of probabilistic algorithms to train a computational model to make predictions ${ }^{13}$. ML has great potential to become a valuable clinical tool as it may overcome the time-consuming and subjective shortcomings of current biomechanical methods, using in diagnosis, treatment optimization, and prediction of outcomes ${ }^{14,15}$. It has already been used to elucidate the underlying biological processes related to knee $O A^{16,17}$. At present, the machine learning methods for the diagnosis of knee OA mainly include support vector machine, Convolutional Neural Network, artificial neural network, etc. However, the diagnostic test accuracy of different machine learning methods is still controversial.

The aim of this systematic review is to gather the existing diagnostic accuracy test research on the use of different machine learning methods and assess their application for detection in screening patients with knee osteoarthritis. Strengths and limitations of the current studies as well as gaps in the research will be highlighted, to uncover elements that could impede this implementation.

\section{Materials And Methods}

\section{1 study registration}

This systematic review has been registered on PROSPERO (registration number: CRD: 42019133305). This research protocol has been developed according to the Preferred Reporting Items for Systematic reviews and Meta-Analyses Protocol (PRISMA-P $)^{18}$, and we will conduct the systematic review and meta-analysis according to the Preferred Reporting Items for a Systematic Review and Meta-analysis of Diagnostic Test Accuracy Studies (PRISMA-DTA) guidelines ${ }^{19,20}$.

\subsection{Search strategy}


The electronic databases PubMed, Cochrane library, EMBASE, and Web of Science will be searched. The keywords will use in the search strategy included ("Osteoarthritis, Knee" or "goneitis" or "knee arthritis" or "articulatio genus" or "gonarthritis") and ("Artificial intelligence" or "deep learning" or "Machine Intelligence" or "machine learning" or "supervised machine learning" or "neural networks" or "data mining" or "Computational Intelligence" or "Al") and ("sensitivity" or "specificity"). Furthermore, reference lists of the included studies will be screened manually for relevance to identify potential studies missed in the systematic search ${ }^{21}$. The search is limited to English language articles, and we did not restrict the study to source, country or publication date. We will provide specific search strategy sample of PubMed and will be shown in supplement 1 .

\subsection{Selection criteria}

\subsubsection{Types of studies}

We will include studies that investigated diagnosis and prediction accuracy of different machine learning methods for Knee Osteoarthritis, the study design will be limited to prospective studies or retrospective studies.

\subsubsection{Types of participants}

Participants suffered from knee OA (clinically diagnosed by the critical of American Rheumatism Association or the KL scale and Clinician's diagnosis) will be included regardless of the patient's race, gender, age, country, disease duration or disease severity. And the patients did not receive an intraarticular knee injection in the one month prior to the study and they had no hip or ankle arthritis.

\subsubsection{Types of outcome measures}

We will assess the following outcomes at the end of the treatment period. The study reporting at least one of the following outcomes will be included. The primary outcome includes the accuracy, sensitivity, specificity. The secondary outcomes consist of Positive predictive value (PPV), Negative predictive value (NPV), false positive rate (FPR), and false detection rate (FDR) and area under the receiver operating characteristic curve (AUC).

\subsection{Exclusion criteria}

We will exclude the studies if: (a) not relevant subject outcome, (b) not methods machine learning, (c) the information provided in the results was insufficient for data extraction, (d) duplicate studies, commentaries, summaries, editorials, letters, or case reports.

\subsection{Study selection}

Two authors (ZL and MT) independently screen a titles and abstracts based on the inclusion or exclusion criteria, record reasons for exclusion of the ineligible studies. And subsequently retrieved all relevant fulltext articles for suitability assessment. If necessary, any discrepancies regarding inclusion will resolved 
through discussion or by consulting a third member of (ZY) the review team until consensus is reached. We will record selection process in sufficient detail to complete a PRISMA flow chart.

\subsection{Data extraction}

Data extraction should be informed by TRIPOD using the TRIPOD adherence guidance, PROBAST and the Checklist for critical Appraisal and data extraction for systematic Reviews of prediction Modelling Studies. We will extract data for qualitative analysis with qualitative analysis from the included studies. For each of the included studies, two reviewers will extract the following information that satisfied the inclusion criteria according to the pre-designed Excel form, Including :(1) basic character of the research object (authors, year of publication, country, patient ages, study design (prospective or retrospective), patient numbers, Body mass index), (2) general dataset (index test, Size of Dataset/images, Stage of clinical diagnosis, reference standard, type of Machine Learning), number of predictors (candidate and final), internal validation type, predictive performance measures (discrimination and calibration), number of models developed and the details of the ML technique used to develop each model (eg, technique, preprocessing, data cleaning, optimization algorithm, predictors selection, penalisation techniques, hyperparameters, code, data availability and so on). Moreover, the TP, TN, FP, and FN results of MRI and PET/CT of each included studies will be included, all the included studies will be analyzed using a $2 \subseteq 2$ contingency table. When available data is insufficient, we plan to contact the authors of included studies to obtain additional information. If we cannot achieve those data, then we will just analyze the available data and also discuss its potential impacts. Disagreements will be discussed and resolved by consensus among the third review authors.

\subsection{Assessment of risk of bias in included studies}

Risk of bias assessment for the systematic review will performed independently by two reviewers (ZY and MT) using PROBAST tool (Prediction model Risk of Bias Assessment Tool) ${ }^{22}$, which evaluates four key domains: patient selection, predictors, outcome and analysis. Each domain is assessed based on the risk of bias and concerns about the applicability to the review (first three domains only). Each question is answer with "yes", "no", "unclear", the level of risk of bias can be judged as "low risk" "high risk" "unclear risk" homologous ${ }^{23}$.

\subsection{Data synthesis and analysis}

Although the included studies fulfill all inclusion criteria, there are still some underlying differences in regard to Stage of clinical diagnosis, target condition and reference standard. If data provided in the paper was sufficient and have sufficiently homogenous for analysis, we will present a quantitative analysis, and included studies will be analyzed using a $2 \times 2$ contingency table, using these tables, numerical values for sensitivity and specificity were obtained from false negative (FN), false positive (FP), true negative (TN) and true positive (TP). If some of the studies do not directly give all the data in the $2 \times 2$ table, we will use the calculator in Review Manager 5.3 to calculate the missing data based on the existing data in the text or the appendix in each study. A descriptive forest plot and summary receiver operating characteristic (SROC) curves will be derived by Review Manager 5.3, the area under the curve 
(AUC) is the final comparison indicator. The criteria for AUC classification are 0.90-1 (excellence), 0.800.90 (good), $0.70-0.80$ (fair), $0.60-0.70$ (poor) and 0.50-0.60 (failure).

We will combine data using a fixed-effect or a random-effects model, on the basis of the clinical or methodological diversity for heterogeneity ${ }^{24}$. If there is no significant statistical heterogeneity is found from the included trials or no data can be combined, we use a fixed effects model to analyze the data. If we find substantial heterogeneity ( $\geq 50 \%$ ), we will use a random-effects model. In addition, different diagnostic thresholds of included studies may lead to heterogeneity, we will use the spearman correlation coefficients to test whether there is a threshold effect. When there is a threshold effect, sensitivity and specificity will be negatively correlated, and the results will present a "shoulder-arm" point distribution on the SROC curve.

For outcomes where we cannot provide a quantitative analysis, we will present the results of individual studies in a narrative synthesis (qualitative analysis). We will resolve any disagreement through discussion among the review authors.

\subsection{Assessment of heterogeneity}

Heterogeneity among the studies will be evaluated using the $R$ statistic. $R>50 \%$ indicates a significant heterogeneity, and under this circumstance, subgroup analysis, influence analysis, as well as metaregression analysis will be performed to ascertain the potential causes from clinical or methodologic heterogeneity ${ }^{25}$. The choice of random effect model or fixed effect model will depend on whether there exists heterogeneity or not. If heterogeneity exists but cannot be explained reasonably and consequently meta-analysis is unavailable to be conducted, we will describe the data.

\subsection{Subgroup analysis}

Owing to differentiation of situation such as age, gender, disease condition, kinds of machine learning method and other unpredictable factors, we will conduct subgroup analysis base on the data to detect the source of heterogeneity.

\subsection{Quality of evidence rating}

Grading of Recommendations Assessment, Development and Evaluation (GRADE) tool will be used to evaluate the overall strength of the evidence ${ }^{26-28}$. Its results will be summarized in tables of Summary of Findings.

\section{Discussion}

This review is the first to apply a systematic approach to evaluate the diagnostic performance of machine learning method in patients with knee OA as compared to existing reference standards. As opposed to the previously diagnose methods (such as clinical experts, $\mathrm{X}$-ray), an advantage of using machine learning method includes a substantial reduction in the manpower required for feature 
engineering, since machine learning-algorithms learn to extract features by themselves. Furthermore, it can also find some features of knee lesions in advance, and these features are not well diagnosed by doctors. It may give clinicians more ways to assist patient in relieving knee joint pain and improve the quality of life. However, due to language barriers, only two languages of the trials can be included, other related studies may be missing. Also, Different methods of machine learning and quality of methodologies may increase the risk of heterogeneity.

In summary, this study will generate present evidence of machine learning method for diagnose of patients with knee $O A$, and will help to reduce the uncertainty about the accuracy of diagnose and prediction. The findings of this study will encourage further suggestions for clinicians or guideline, and will draw wide attention for both patients and researchers.

\section{Declarations}

\section{Acknowledgments}

Not applicable.

\section{Author contributions}

Y Zhou: planned and designed the research; Y Zhou and T Mu: project development, data collection, analysis and interpretation, manuscript writing, article revised; $\mathrm{T} \mathrm{Mu}$ : data collection, analysis and interpretation, manuscript writing; XC Kong: data collection, analysis and interpretation; Le Zhang: academic oversight and edited all drafts, article revised

All authors critically revised the Article for important intellectual content and approved the final version of the manuscript.

\section{Funding}

This work was supported by Teaching Research and Reform Foundation of Gansu University of Chinese Medicine (YBXM-16) The funding organizations had no role in the design and conduct of this systematic review, or in the preparation of, or decision to submit, the protocol.

\section{Availability of data and materials}

Not applicable.

\section{Ethics approval and consent to participate}

Not applicable.

\section{Consent for publication}

Not applicable. 


\section{Competing interests}

We declare that we have no competing interests.

\section{References}

1. Peat G, McCarney R, Croft P. Knee pain and osteoarthritis in older adults: a review of community burden and current use of primary health care. Annals of the rheumatic diseases. 2001;60(2):91-7.

2. Cross M, Smith E, Hoy D, et al. The global burden of hip and knee osteoarthritis: estimates from the global burden of disease 2010 study. Annals of the rheumatic diseases. 2014;73(7):1323-30.

3. Murphy L, Schwartz TA, Helmick CG, et al. Lifetime risk of symptomatic knee osteoarthritis. Arthritis rheumatism. 2008;59(9):1207-13.

4. Moher $D$, Glasziou $P$, Chalmers $I$, et al. Increasing value and reducing waste in biomedical research: who's listening? Lancet. 2016;387(10027):1573-86.

5. Hubertsson J, Petersson IF, Thorstensson CA, Englund M. Risk of sick leave and disability pension in working-age women and men with knee osteoarthritis. Annals of the rheumatic diseases. 2013;72(3):401-5.

6. Karsdal MA, Michaelis M, Ladel C, et al. Disease-modifying treatments for osteoarthritis (DMOADs) of the knee and hip: lessons learned from failures and opportunities for the future. Osteoarthritis cartilage. 2016;24(12):2013-21.

7. Messent EA, Ward RJ, Tonkin CJ, Buckland-Wright C. Tibial cancellous bone changes in patients with knee osteoarthritis. A short-term longitudinal study using Fractal Signature Analysis. Osteoarthritis cartilage. 2005;13(6):463-70.

8. Richmond J, Hunter D, Irrgang J, et al. Treatment of osteoarthritis of the knee (nonarthroplasty). The Journal of the American Academy of Orthopaedic Surgeons. 2009;17(9):591-600.

9. Altman R, Asch E, Bloch D, et al. Development of criteria for the classification and reporting of osteoarthritis. Classification of osteoarthritis of the knee. Diagnostic and Therapeutic Criteria Committee of the American Rheumatism Association. Arthritis rheumatism. 1986;29(8):1039-49.

10. Menashe L, Hirko K, Losina E, et al. The diagnostic performance of MRI in osteoarthritis: a systematic review and meta-analysis. Osteoarthritis cartilage. 2012;20(1):13-21.

11. Karachalios T, Zibis A, Papanagiotou P, Karantanas AH, Malizos KN, Roidis N. MR imaging findings in early osteoarthritis of the knee. European journal of radiology. 2004;50(3):225-30.

12. Hortobagyi T, Westerkamp L, Beam S, et al. Altered hamstring-quadriceps muscle balance in patients with knee osteoarthritis. Clin Biomech (Bristol Avon). 2005;20(1):97-104.

13. Jordan MI, Mitchell TM. Machine learning: Trends, perspectives, and prospects. Science (New York NY). 2015;349(6245):255-60.

14. Manlhiot $\mathrm{C}$. Machine learning for predictive analytics in medicine: real opportunity or overblown hype? European heart journal cardiovascular Imaging. 2018;19(7):727-8. 
15. Ghahramani Z. Probabilistic machine learning and artificial intelligence. Nature. 2015;521(7553):452-9.

16. Ashinsky BG, Coletta CE, Bouhrara M, et al. Machine learning classification of OARSI-scored human articular cartilage using magnetic resonance imaging. Osteoarthritis cartilage. 2015;23(10):1704-12.

17. Heard BJ, Rosvold JM, Fritzler MJ, El-Gabalawy H, Wiley JP, Krawetz RJ. A computational method to differentiate normal individuals, osteoarthritis and rheumatoid arthritis patients using serum biomarkers. Journal of the Royal Society Interface. 2014;11(97):20140428.

18. Shamseer $L$, Moher $D$, Clarke $M$, et al. Preferred reporting items for systematic review and metaanalysis protocols (PRISMA-P) 2015: elaboration and explanation. BMJ. 2015;350:g7647.

19. Mclnnes MDF, Moher D, Thombs BD, et al. Preferred Reporting Items for a Systematic Review and Meta-analysis of Diagnostic Test Accuracy Studies: The PRISMA-DTA Statement. Jama. 2018;319(4):388-96.

20. Li Y, Cao L, Zhang Z, et al. Reporting and methodological quality of COVID-19 systematic reviews needs to be improved: an evidence mapping. Journal of clinical epidemiology. 2021;135:17-28.

21. Tudor Car L, Li L, Smith H, Atun R. Cochrane review: Search strategies to identify observational studies in MEDLINE and EMBASE. Journal of evidence-based medicine. 2019;12(3):225-6.

22. Wolff RF, Moons KGM, Riley RD, et al. PROBAST: A Tool to Assess the Risk of Bias and Applicability of Prediction Model Studies. Annals of internal medicine. 2019;170(1):51-8.

23. Yan P, Yao L, Li H, et al. The methodological quality of robotic surgical meta-analyses needed to be improved: a cross-sectional study. Journal of clinical epidemiology. 2019;109:20-9.

24. Yang K. Evidence-based social science: the origin, development and prospects. Libr Inf 2018;3:1e10.. 2018.

25. Tian J, Zhang J, Ge L, Yang K, Song F. The methodological and reporting quality of systematic reviews from China and the USA are similar. Journal of clinical epidemiology. 2017;85:50-8.

26. Guyatt GH, Oxman AD, Vist GE, et al. GRADE: an emerging consensus on rating quality of evidence and strength of recommendations. BMJ. 2008;336(7650):924-6.

27. Norris SL, Meerpohl JJ, Akl EA, et al. The skills and experience of GRADE methodologists can be assessed with a simple tool. Journal of clinical epidemiology. 2016;79:150-8.e151.

28. Yao L, Sun R, Chen YL, et al. The quality of evidence in Chinese meta-analyses needs to be improved. Journal of clinical epidemiology. 2016;74:73-9.

\section{Supplementary Files}

This is a list of supplementary files associated with this preprint. Click to download.

- PRISMAPchecklist.doc

- Supplement1.docx 